\title{
A gene expression profile related to immune dampening in the tumor microenvironment is associated with poor prognosis in gastric adenocarcinoma
}

\author{
Fatima Solange Pasini • Bruno Zilberstein • Igor Snitcovsky • Rosimeire Aparecida Roela • \\ Flavia R. Rotea Mangone • Ulysses Ribeiro Jr. • Suely Nonogaki • Glauber Costa Brito • \\ Giovanna D. Callegari - Ivan Cecconello • Venancio Avancini Ferreira Alves • \\ José Eluf-Neto $\cdot$ Roger Chammas $\cdot$ Miriam Hatsue Honda Federico
}

Received: 21 November 2012/Accepted: 17 October 2013/Published online: 12 November 2013

(C) The Author(s) 2013. This article is published with open access at Springerlink.com

\begin{abstract}
Background The TNM Classification of Malignant Tumours (TNM) staging system is the primary means of determining a prognosis for gastric adenocarcinoma (GC). However, tumor behavior in the individual patient is unpredictable and in spite of treatment advances, a classification of 'advanced stage' still portends a poor prognosis. Thus, further insights from molecular analyses are needed for better prognostic stratification and determination of new therapeutic targets.

Methods A total of fifty-one fresh frozen tumor samples from patients with histopathologically confirmed diagnoses of GC, submitted to surgery with curative intent, were included in the study. Total RNA was extracted from an initial group of fifteen samples matched for known prognostic factors, categorized into two subgroups, according to patient overall survival: poor ( $<24$ months) or favorable (at or above 24 months), and hybridized to Affymetrix
\end{abstract}

Electronic supplementary material The online version of this article (doi:10.1007/s00535-013-0904-0) contains supplementary material, which is available to authorized users.

F. S. Pasini · I. Snitcovsky · R. A. Roela · R. Chammas · M. H. H. Federico

Departamento de Radiologia e Oncologia, Faculdade de

Medicina, Universidade de São Paulo, São Paulo, Brazil

F. S. Pasini · I. Snitcovsky ( () ) F. R. R. Mangone ·

G. C. Brito · R. Chammas

Centro de Investigação Translacional em Oncologia, Instituto de

Câncer do Estado de São Paulo (ICESP), Av. Dr. Arnaldo, 251,

$8^{\circ}$ andar, Cerqueira César, São Paulo, SP 01246-000, Brazil

e-mail: igor@lim24.fm.usp.br; fatima@lim24.fm.usp.br

B. Zilberstein · U. Ribeiro Jr. · I. Cecconello

Disciplina de Cirurgia do Aparelho Digestivo, Faculdade de

Medicina, Universidade de São Paulo, São Paulo, Brazil
Genechip human genome U133 plus 2.0 for genes associated with prognosis selection. Thirteen genes were selected for qPCR validation using those initial fifteen samples plus additional thirty-six samples.

Results A total of 108 genes were associated with poor prognosis, independent of tumor staging. Using systems biology, we suggest that this panel reflects the dampening of immune/inflammatory response in the tumor microenvironment level and a shift to Th2/M2 activity. A gene trio (OLRI, CXCL11 and ADAMDEC1) was identified as an independent marker of prognosis, being the last two markers validated in an independent patient cohort.

Conclusions We determined a panel of three genes with prognostic value in gastric cancer, which should be further investigated. A gene expression profile suggestive of a dysfunctional inflammatory response was associated with unfavorable prognosis.

Keywords Biomarkers of prognosis - Immune response $\cdot$ Inflammatory response $\cdot$ Gastric cancer

U. Ribeiro Jr.

Coordenação Cirúrgica, Instituto de Câncer do Estado de São

Paulo (ICESP), São Paulo, Brazil

S. Nonogaki

Divisão Central de Patologia, Laboratório de Imuno-

histoquímica, Instituto Adolfo Lutz, São Paulo, SP, Brazil

G. D. Callegari - V. A. F. Alves

Departamento de Patologia, Faculdade de Medicina,

Universidade de São Paulo, São Paulo, Brazil

J. Eluf-Neto

Departamento de Medicina Preventiva, Faculdade de Medicina,

Universidade de São Paulo, São Paulo, Brazil 


\section{Introduction}

The major indicator of prognosis in patients with stomach adenocarcinoma after curative surgical treatment is the TNM stage category. Stage I, in general, portends a good prognosis, in contrast to stages IIIb/IV, and II/IIIa, which are associated with a poor and intermediate prognosis, respectively. Additional prognostic factors include the histological subtype and the tumor location [1]. The clinical course, however, is strikingly unpredictable in the individual patient, which strongly suggests that there are still unknown biological determinants of tumor behavior.

Several studies have addressed the prognostic significance of molecular alterations in gastric cancer, such as vascular endothelial growth factor (VEGF), placental growth factor (PlGF) [2, 3] and others factors that control cell growth, such as p27 or cyclin E [4, 5], oncogenes (c-erbB2 and c-myc), and tumor suppressor genes including p53 [6]. However, there are confounding results among different studies and markers, minimizing the information about the individual prognosis [7].

The biological complexity of gastric cancer indicates that the overall molecular evaluation of a tumor in an individual patient can be a more interesting strategy for the identification of biomarkers. With the technological advancements in the last decade, it became feasible to evaluate gene expression profiles by cDNA microarrays or qPCR (polymerase chain reaction in real time). Chen et al. [8] presented a predictive model based on three genes derived from a gene expression study of eighteen paired patient samples (primary tumor and adjacent mucosa). In 2007, Marchet et al. [9] proposed a model based on three genes that were predictive of lymph node involvement by tumor cells, using a cohort of thirty-two patients with primary gastric carcinoma. Recently, the expression of a selection of eighty-four known human genes, representing six biological pathways involved in transformation and tumorigenesis, were determined by qPCR in forty-eight gastric cancer primary tumors, resulting in a predictive model for survival consisting of four genes [10]. Despite shedding little light on gastric cancer biology, these models are intriguingly composed of non-overlapping genes. In this study, our aim was to generate a molecular signature of gastric cancer with prognostic value, which could contribute to understanding of the mechanism underlying an aggressive clinical course. A panel of one hundred and eight genes was associated with poor prognosis, independent of tumor staging. Using a systems biology approach, we suggest that this panel reflects a dampening of the immune/inflammatory responses at the level of the tumor microenvironment. In addition, three genes (OLR1, CXCL11 and $A D A M D E C 1$ ) were identified as independent markers of prognosis, and partially validated in silico in an independent cohort.

\section{Methods}

Patients and tissue samples

The study was approved by the Internal Review Board of the Hospital das Clínicas da Faculdade de Medicina da Universidade de São Paulo (No. 222/01). After written informed consent was received from each patient, we collected tumor specimens and clinical data from fifty-one gastric adenocarcinoma patients who underwent radical gastrectomy with potentially curative intent, with D2 lymph node dissection (more than 25 retrieved lymph nodes) as a primary treatment option. The tumor samples were collected at the time of surgical treatment, followed by fresh freezing in liquid nitrogen and storage at $-80{ }^{\circ} \mathrm{C}$. Stage of gastric cancer was classified according to the 6 th edition of UICC (TNM) classification system, as recommended also by the American Joint Committee on Cancer. Histopathological diagnosis was reviewed and confirmed in all cases by the team of pathologists led by one of the authors (VAFA).

Overall survival was calculated from the date of primary surgery to the date of last follow-up or to the date of death. In our series, $58.8 \%$ of patients were male, with a predominance of the diffuse histological type (70.6\%) and stage III/ IV (74.5\%). The median follow-up was 29.8 months, and at the last follow-up, 16 patients were still alive. Regarding smoking, $60.7 \%(31 / 51)$ of patients were active smokers or had previously used tobacco, and $43.1 \%$ reported alcohol consumption (Table 1, Supplemental Table 1).

\section{Total RNA extraction from tumor tissues}

Total RNA was extracted using the Trizol reagent (Invitrogen Life Technologies) and fifteen micrograms were purified using the RNeasy mini kit (Qiagen, Germany) according to the manufacturer's protocols. RNA concentration was determined by NanoDrop ND-1000 UV-Vis Spectrophotometer (NanoDrop Technologies, Wilmington, DE, USA). Purity and integrity (28S/18S ratio) were assessed by RNA 6000 nano assay chips using an Agilent Bioanalyzer Model 2100 (Agilent Technologies, CA, USA). Only samples with an RNA integrity number (RIN) higher than 6.0 (Agilent software) were included in the analysis.

Microarray assay and data analysis

Fifteen samples were categorized into two groups, according to patient overall survival: poor $(<24$ months $)$ or favorable (24 months or above). The samples were matched for known prognostic factors, including tumor TNM stage, histological subtype, and patient gender. Five micrograms of total 
Table 1 Clinical and pathological characteristics of 51 patients with gastric adenocarcinoma

\begin{tabular}{ll}
\hline Characteristics & Total \\
\hline Age & \\
Median (range) & 66 (37-89) \\
Gender & 21 \\
Female & 30 \\
Male & \\
Tumor type & 36 \\
Diffuse & 10 \\
Intestinal & 2 \\
Mixed & 3 \\
ND & \\
TNM stage & 13 \\
I/II & 38 \\
III/IV & \\
Habits & \\
Smoking & \\
Yes & 13 \\
Stopped & 18 \\
Never & 20 \\
Consumption of alcohol & \\
Yes & 10 \\
Stopped & 29 \\
Never & \\
\hline
\end{tabular}

purified RNA from each sample were processed for microarray analysis, in accordance with Affymetrix protocol, using One Cycle Labeling Kit (Affymetrix, Santa Clara, CA, USA). Briefly, purified RNA was used to synthesize cDNA. Afterwards, Biotin-labeled cRNA was synthesized from double-strand cDNA using an IVT labeling kit (Affymetrix, Santa Clara, CA, USA). Quality and concentration were assessed using a NanoDrop and 2100 Bioanalyzer. Fifteen micrograms of biotinylated cRNA were fragmented and hybridized onto the Genechip Human Genome U133 plus 2.0 array (Affymetrix, 54,210 probe sets covering over 47,000 transcripts and splice variants) and scanned with Affymetrix GeneChip Scanner 3000. After image acquisition, raw fluorescent signal (cel. file) from Affymetrix GeneChip Operating Software (GCOS) was assessed with R Bioconductor software and the normalization was performed using Robust Multichip Analysis (RMA). Limma and Rank Product analyses with R Bioconductor software were used to identify differentially expressed genes between the groups of poor and favorable prognosis.

Unsupervised hierarchical clustering based on Euclidean distance and complete linkage was performed using differentially expressed genes. The reliability of the clustering was assessed by the bootstrap technique implemented in TMEV software. The function of the differentially expressed genes was mapped to the biologic process and pathways, based on Gene Ontology (GO) and Kyoto Encyclopedia of Genes and Genomes (KEGG) pathway databases, using the FunNet 1.00-9 software (http://www.funnet.info), where the $P$ value is the significance of the gene enrichment of the considered GO Biological Process category or annotation cluster, calculated with a unilateral Fisher's exact test.

The array data have been submitted to the National Center for Biotechnology Information (NCBI) Gene Expression Omnibus (GEO) and are accessible through GEO series accession no. GSE38749 (http://www.ncbi. nlm.nih.gov/geo/).

Quantitative reverse transcription (RT)-PCR (qRT-PCR)

Reverse transcription reactions were performed with Super Script III-First Strand Synthesis Super Mix (Invitrogen Life Technologies) using $0.05 \mu \mathrm{g} / \mu \mathrm{L}$ random hexamer primers. cDNA samples $(25-50 \mathrm{ng})$ were subjected to qPCR assays in duplicate using SYBR Green methodology with the Power SYBR ${ }^{\circledR}$ Green PCR Master Mix (Applied Biosystems, Life Technologies), followed by Rotor-Gene 6 System software analysis (Corbett Research, Mortlake, Australia). Gene-specific primers were designed using the Primer 3 software (http://frodo.wi.mit.edu/primer3/) to generate a PCR product in the $3^{\prime}$ portion, spanning the translated region of the target mRNA (Supplemental Table 2). Sequences present in different exons, preferentially separated by long introns, were selected, according to sequences deposited at http://www.ncbi.nlm.nih.gov/ nucleotide. To avoid non-specific product formation, BLAST analysis (http://www.ncbi.nlm.nih.gov/blast) was carried out. For all primers used, the reactions conditions were: $95^{\circ} \mathrm{C}$ for $10 \mathrm{~min}$, followed by 40 cycles at $95^{\circ} \mathrm{C}$ for $15 \mathrm{~s}$ and in annealing temperature of $59{ }^{\circ} \mathrm{C}$ for $60 \mathrm{~s}$.

Four housekeeping genes (ACTB, GUSB, HPRT1 and $R P L P O)$ were tested in all samples (data not shown). The expression of GUSB ( $\beta$-Glucuronidase), RPLPO (Large ribosomal protein), and HPRT1 (Hypoxanthine phosphoribosyl-transferase I) showed lower variation among the samples. The expression of these genes was then used to calculate a normalization factor (NF) for each sample analyzed, using the geNorm software tool [11] (available at http://medgen.ugent.be/ jvdesomp/genorm/). Relative gene expression of the target genes was then normalized using the $\left[\left(E_{\text {target }}+1\right)^{\Delta C_{\text {t target }}}\right] / \mathrm{NF}$ expression [12].

In silico validation of the gene expression signature

To validate our results in a larger and independent patient cohort, we replicated the bioinformatics analysis on 
publically available datasets. Two sets of criteria to select the datasets were used: (1) the gene expression profile should be derived from the Affymetrix Human Genome U133A or U133 Plus 2.0 Array, and (2) the time of overall survival of each patient should be available. We searched in the Gene Expression Omnibus (GEO) repository (http:// www.ncbi.nlm.nih.gov/geo) and ArrayExpress (http:// www.ebi.ac.uk/arrayexpress) to find datasets satisfying these criteria and we used the search string ((gastric cancer) AND CEL[Supplementary Files] AND (GPL96[ACCN] OR GPL570[ACCN]) AND tumor[Sample Source]) with two filters: organism (Homo sapiens) and study type (expression profile array). We manually checked whether dataset clinical outcomes were available, if tumor samples were from operable patients with gastric adenocarcinoma, and if the cel. files were available. RankProd and Limma analyses, with the same parameters used in our original study, were performed to define the differentially expressed genes. The function of the genes was mapped to biological processes and pathways, also based on Gene Ontology (GO) and Kyoto Encyclopedia of Genes and Genomes (KEGG) pathway databases.

\section{Immunohistochemical staining}

The assessment of immunoexpression of CXCL11 was performed with polyclonal antibody chicken anti-human (Lifespan Biosciences, cat\# LS-C96091, Seattle, Wa, USA), diluted 1:100 and incubated overnight at $4{ }^{\circ} \mathrm{C}$. Antigen retrieval was performed by pressure cooker (Rochedo, A La Carte) using sodium citrate buffer pH 6.0. Endogenous peroxidase was quenched with $6 \%$ hydrogen peroxide. Endogenous biotin was blocked with Biotin Blocking System, as instructed by the manufacturer (Dako cat\# x0590, Carpinteria, CA, USA). Slides were washed with phosphate-buffered saline and incubated with the secondary biotinylated antibody, goat polyclonal to chicken IgY-H\&L (Abcam, cat\#ab97133, Cambridge, MA, USA), diluted 1:100 and amplified with ABC system (Vectastain Elite HRP, cat\# PK6100, Burlingame, CA, USA), diluted 1:100. Sections were developed with $3,3^{\prime}$ diaminobenzidine (Sigma, cat\# D5637, St Louis, MO, USA) and counterstained with hematoxylin.

The assessment of immunoexpression of OLR1 was performed with polyclonal antibody rabbit anti-human (Abcam cat \# ab85839, Cambridge, MA, USA), diluted 1:600 and incubated overnight at $4{ }^{\circ} \mathrm{C}$. Antigen retrieval was performed by pressure cooker (Rochedo, A La Carte) using Tris (10 mM)-EDTA $(1 \mathrm{mM})$ buffer. Endogenous peroxidase was quenched with $6 \%$ hydrogen peroxide. Slides were washed with phosphate-buffered saline and incubated with the primary antibody enhancer (UltraVision LP Value Large Volume Detection System HRP Polymer,
Thermo Scientific, cat\# TL-125-HLS) followed by Polymer labeled with HRP. Sections were developed with $3,3^{\prime}$ diaminobenzidine (Sigma, cat\# D5637, St Louis, MO, USA) and counterstained with hematoxylin. Stained sections were evaluated by one blinded pathologist. Immunohistochemical staining for the proteins was evaluated by a semi-quantitative scoring system: $0=$ negative staining; $1+$, faintly $=<5 \%$ stained cells; $2+$, moderate $=6-25 \%$ stained cells, $3+$, strong $=25-50 \%$ and $4+$, very strong $=>51 \%$ stained cells.

Statistical and bioinformatics analysis

RankProd and Limma analyses, provided by the R Bioconductor program, were employed to analyze microarray data from patient samples with poor survival ( $<24$ months) or favorable survival ( $\geq 24$ months). Differentially expressed genes were those satisfying a significance level $<0.05$ (two-sided) and FDR (false discovery rate) considering 1,000 permutations. Relevance networks were constructed as indicated, considering Pearson's correlation, $P<0.05$.

For RT-qPCR experiments, the Shapiro-Wilk test was performed to determine the distribution of the relative normalized expression data.

The level of significance of the differential gene expression among clinicopathological parameters was determined by the Mann-Whitney test, and a two-sided $P$ value $\leq 0.05$ was considered to be significant. Overall survival analyses were performed using the Kaplan-Meier method and compared by the log-rank test. To generate a risk score with the selected genes, we adopted a previously established strategy by using the Cox regression coefficient of each gene [10]. Multivariate analysis was conducted to evaluate the prediction accuracy of our survival profile in comparison with the standard clinicopathological covariates by Cox proportional hazards regression using SPSS $11.0 \mathrm{v}$ software (Statistics Packet for Social Sciences, Chicago. IL, USA).

\section{Results}

The identification of the prognostic gene panel was accomplished by grouping patient samples and the procedures described in supplemental Fig. 1.

Gene selection associated with prognosis

Data from seven samples from patients with favorable prognoses were compared to eight samples from patients with poor prognoses, matched for known prognostic factors: TNM staging, histological subtype (intestinal/diffuse) 


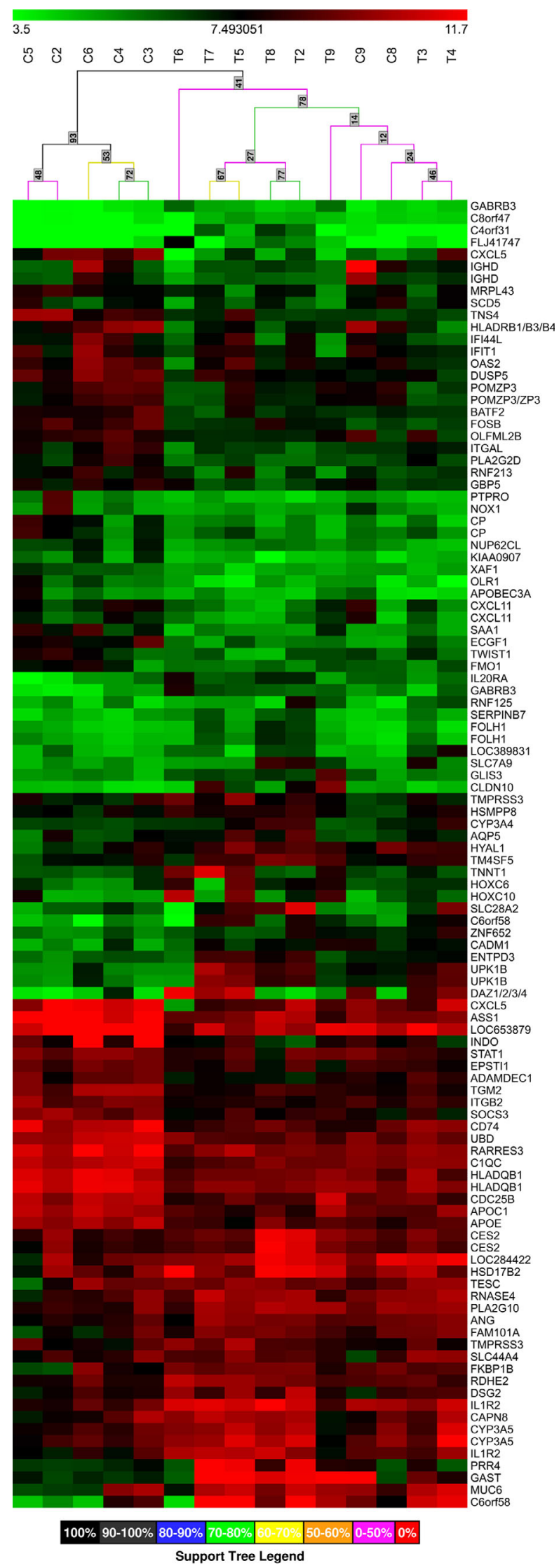

4 Fig. 1 Dendrogram representing the unsupervised hierarchical clustering using Euclidean distance and average linkage as parameters. The genes are arranged in each row, and the samples are in columns. Five samples from patients with good prognosis (C2-C6) were correctly classified, while two samples, C8 and C9, were misclassified, together with samples from patients with a poor prognosis (T2-T9)

and gender. After performing statistical analyses, 248 probes were identified by using the Limma method, considering $P<0.05$ and an expression ratio of poor/favorable improvement, \pm 1.7 . The RankProd method identified 445 probes differentially expressed with a false discovery rate $($ FDR $)<0.05$. Then, the 118 probes identified in common by both methods were recorded and identified a total of 108 genes. Of these, 54 probes showed a decreased expression, always considering the relationship between poor/favorable prognosis, which identified 48 unique genes.

Among the probes with increased expression, 46 unique genes were annotated. In order to verify whether the genes found in each comparison would really be able to distinguish the samples according to the prognoses of patients (poor vs. favorable), a hierarchical clustering was performed using the values of normalized signal intensity of only differentially expressed genes. Five out of seven samples from patients with favorable prognosis were correctly classified, while two samples (C8 and C9) were misclassified, together with samples from patients with a poor prognosis (Fig. 1).

Gene ontology, network and immune gene-related analysis

The most important biological functions of genes differentially expressed between the groups with poor vs. favorable prognosis were determined with the FunNet program. Most genes that were downregulated in the poor prognosis group (Supplemental Table 3), like CXCL5, CXCL11, HLA-DQB1, HLADRB1-5, OLR1 and NOX1, were annotated with biological functions related to immune/inflammatory systems $(43.4 \%)$, while upregulated genes (Supplemental Table 4) were related to cellular catabolic process/oxidative demethylation (66.6\%CYP3A4, CYP3A5) with $P$ value and FDR below 0.05 (Fig. 2).

Pearson correlations between 96 unique differentially expressed genes were then calculated (red line, Fig. 3a, b), using seven samples from the favorable group (Fig. 3a) or eight from the poor prognosis patients (Fig. 3b). To generate a reference distribution of correlations, 96 genes were randomly selected among all genes in the chip and Pearson correlations were calculated in a comprehensive pair-wise manner. This analysis was repeated 100 times, generating reference correlations (blue curve) that showed a 


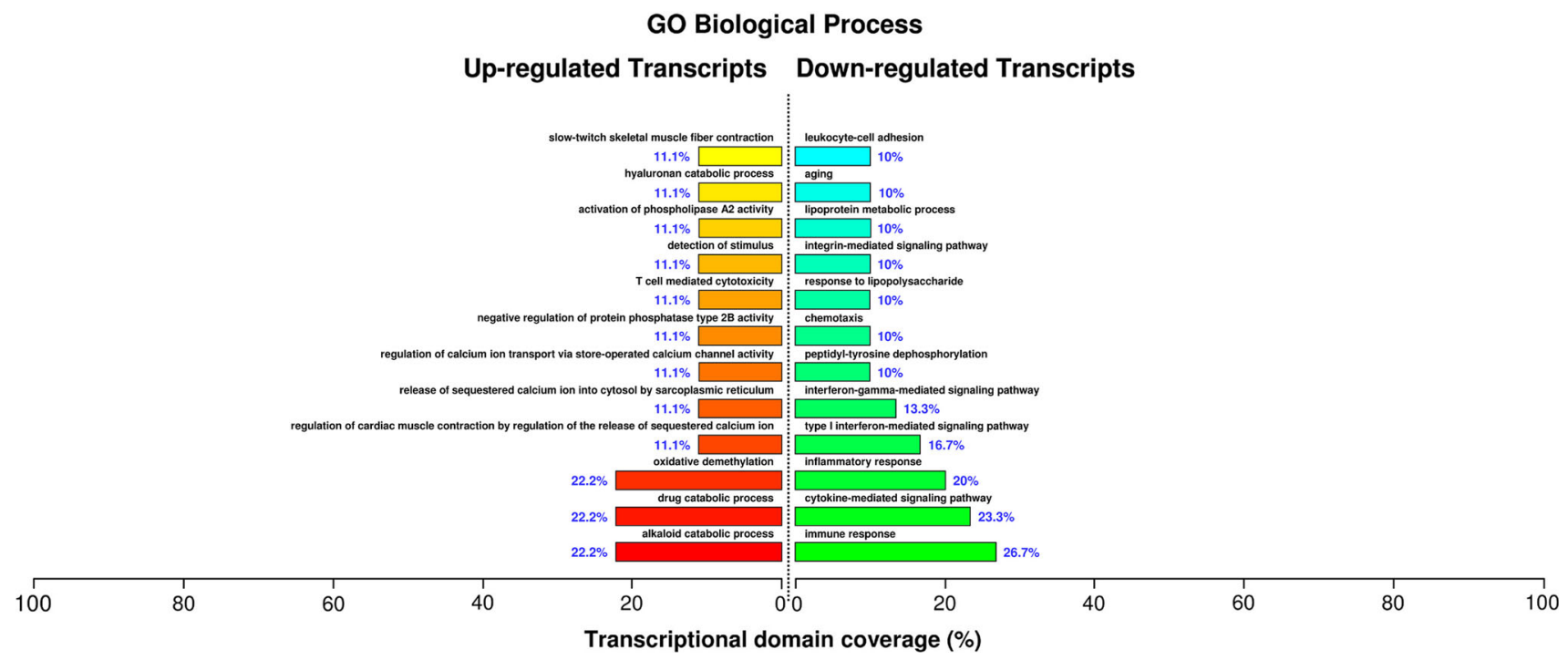

Fig. 2 Graphical representation of the most important biological functions associated with 108 differentially expressed genes identified when comparing tumor samples from gastric adenocarcinoma patients with poor or favorable prognosis

distribution difference compared to the correlations calculated using favorable or poor prognosis samples (Kolmogorov-Smirnov test, $p=3 \times 10^{-41}$ and $p=3 \times$ $10^{-46}$, respectively). Then, we constructed relevance networks (Fig. 3c, d) with all 23 co-regulated genes (Pearson correlation, $P<0.05)$ annotated as involved in immune response. Interestingly, we observed that correlations between these genes tended to be more positive in patients with favorable prognosis than with poor prognosis (Fig. 3a-d). In addition, even with the same set of 23 immune system-related genes, the favorable prognosis network showed more connections (45 edges, Fig. 3c) than the poor prognosis network (23 edges, Fig. 3d). Overall, this greater coordination suggests that the immune system is more actively regulated in patients with favorable prognoses compared to those with a poor outlook.

Gene clustering analysis revealed distinct expression patterns for type 1 helper T cell (Th1)-related gene set, and the patients with poor prognosis were separated from those with favorable prognosis with $75 \%$ accuracy (Supplemental Fig. 2). No associations were found between type 2 helper T cell (Th2), type 17 helper cells (Th17), Regulatory $\mathrm{T}$ cells (Tregs), M1 and M2 classical macrophage-related gene sets and prognosis (data not shown).

Validation of differentially expressed genes by real-time PCR

After annotation of 96 genes and a literature search on the possible associations between the expression of these genes and prognosis of patients with gastric cancer, 13 genes listed among those with the most important biological functions were selected for validation. We selected nine genes (CXCL5, CXCL11, HLADQB1, HLADRB, OLR1, $C A D M 1, C D C 25 B$, ITGAL, ADAMDEC1) with decreased expression and four (MUC6, DAZ, GAST, CLND10) with increased expression in the group of tumors with poor prognosis compared to the favorable prognosis group. The expression of these genes has been successfully determined in fifty-one primary tumors, including those fifteen used in microarray analysis.

No significant differences were found between the expression of any of these markers in relation to sociodemographic features, such as age and alcohol or tobacco consumption. No differences were also found regarding the lymph node status, tumor size, cell differentiation (diffuse or intestinal) and tumor staging. The determination of survival curves was based on the relative expression of genes of interest in tumor tissue, where the tumors were categorized as positive (above) or negative (equal to or below) the cutoff point, optimized by ROC curve, and in some cases the actual median (Supplemental Table 5). Among the genes that showed decreased expression associated with poor prognosis of patients, six out of nine $(66.7 \%)$ were validated. Thus, in univariate analysis, patients with tumors showing negative expression of CXCL11, HLADQB1, ADAMDEC1, ITGAL, CDC25B and OLRl had a worse prognosis compared to patients with tumors expressing these markers (Supplemental Fig. 3). Among the four genes (MUC6, DAZ, CLND10 and GAST) whose increased tumor expression were associated with poor prognosis by microarray assays, only CLND10 and $D A Z$ were further validated (Fig. $4 \mathrm{a}, \mathrm{b}$, respectively). Thus, patients with positive expression of these markers in tumors showed a lower median survival of 22.5 and 24.4 months, respectively, while patients with negative 

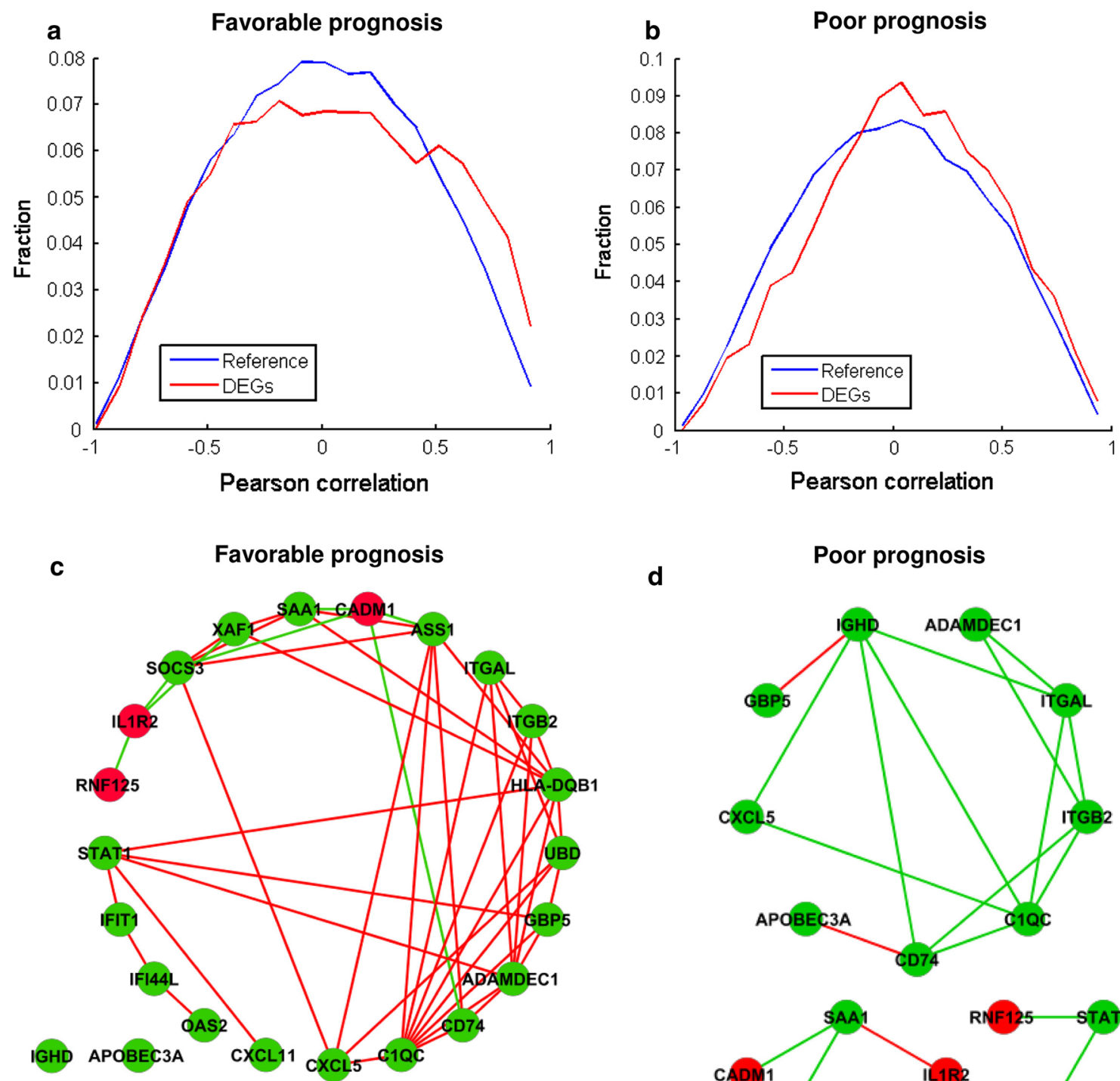

d

Poor prognosis
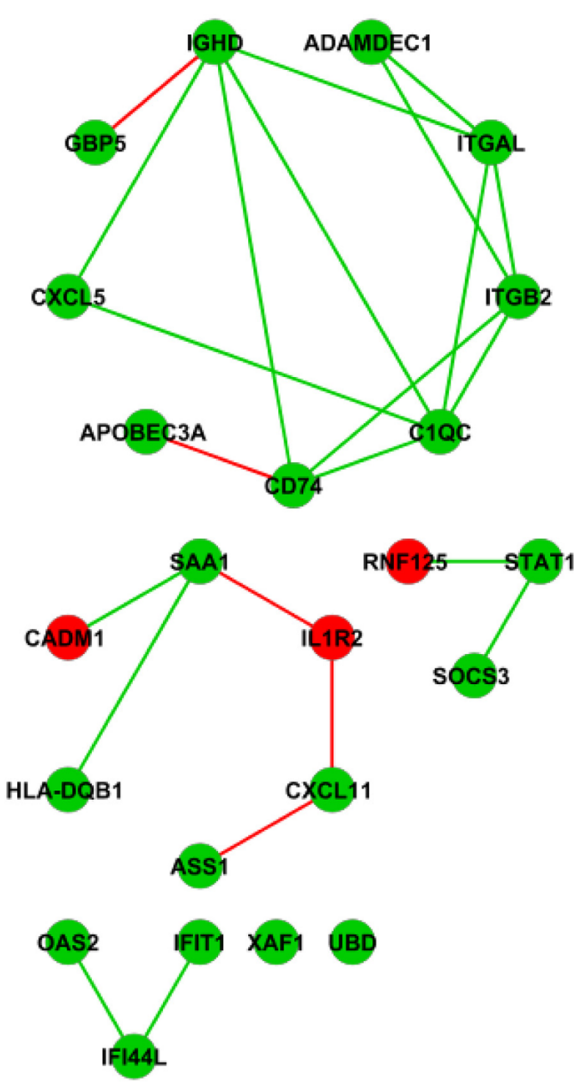

Fig. 3 Distribution of correlations and relevance networks calculated using data from favorable or poor prognosis patients. a, b The correlations between 108 differentially expressed genes (DEGs) (red curve) were compared to a reference dataset generated from random sampling (blue curve). c, d Relevance networks constructed using co-

expression had a median survival of 46.3 and 32.2 months, respectively, although statistical significance was not reached $(P=0.12$ and $P=0.13$, respectively). In relation to MUC6 and GAST genes, no difference in survival expressed DEGs (Pearson correlation, $P<0.05$ ) annotated as involved in global immune response. The favorable prognosis network showed more positive correlations than the poor prognosis network

between groups was observed $(P=0.94$ and $P=0.45$, Log Rank Test, data not shown).

Patients without lymph node involvement ( $\mathrm{pN}$ ) showed a trend toward longer overall survival (pN0: not achieved, 
Fig. 4 Kaplan-Meier curves for overall survival according to tumor expression of mRNA CLND10 (a), and DAZ1-4 (b). The patients were classified as positive (up) or negative (equal to or below) in accordance with the cutoff optimized by the ROC curve. Patients with positive tumor expression for these markers tended to have worse overall survival (Logrank test). Kaplan-Meier curves for overall survival according to tumor expression of the score predictive of survival (c). Patients were classified as having high-risk $(-0.23$ to $-2.09)$ and low-risk ( -33.26 to $-2.10)$ scores according to the joint mRNA expression of CXCL11 and OLR1 in the primary tumor. Patients with high-risk predictive scores had worse overall survival $(P=0.003) . n$ number of patients
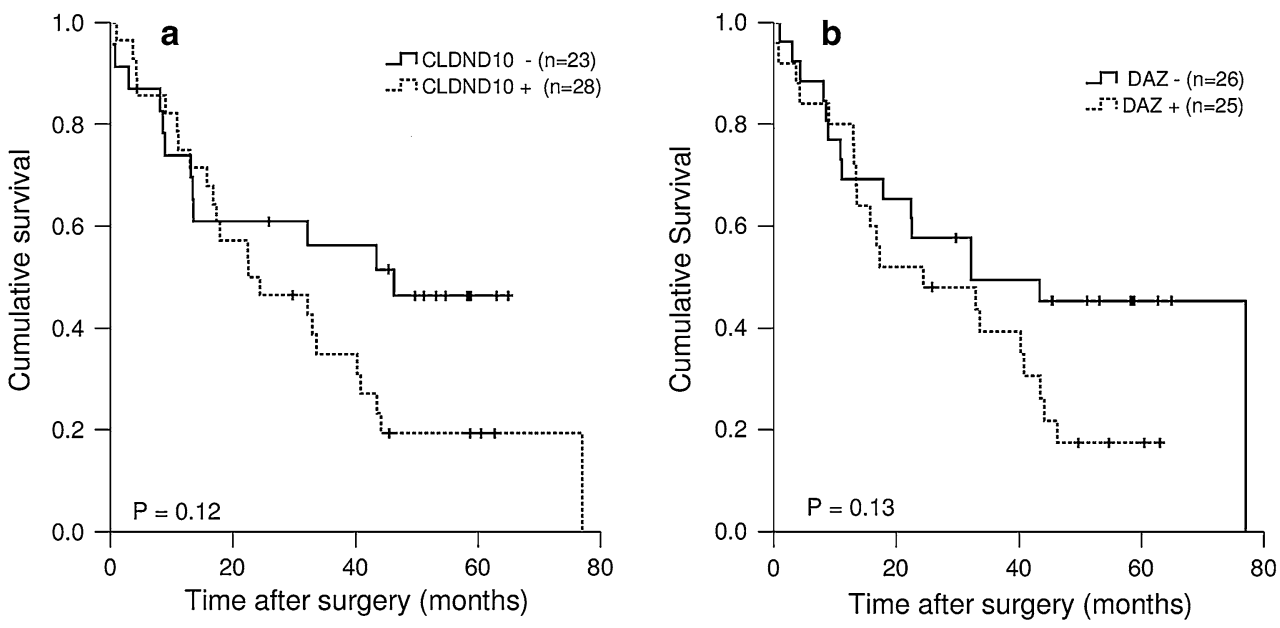

$\mathrm{pN}+: 22.5$ months, $P=0.07)$. Patients with an advanced T stage $(\mathrm{T} 3+\mathrm{T} 4$ : median survival $=17.8$ months $)$ had lower overall survival compared to patients with an early $\mathrm{T}$ stage (T1 + T2: 77.0 months, $P=0.008)$. Additionally, patients with advanced stage (IIIa + IIIb: median $=22.4$ months) tumors had a shorter survival compared to patients with stage $\mathrm{I} / \mathrm{Ib} / \mathrm{II}$ tumors (median $=$ not achieved, $P=0.004$ ).

Predictive model of survival in patients with gastric carcinoma

The model proposed by Xu et al. [10] was used to develop a predictive survival model of patients with gastric carcinoma, regardless of tumor stage. Initially, a COX univariate analysis was performed using the expression data of the 13 genes, with overall survival as a dependent variable. The genes were ordered based on the predictive power of each gene (death risk). Four genes (CXCL11, HLADRB, $O L R 1$, and $A D A M D E C l$ ) were obtained using the conventional risk $\pm 2.0(P<0.05)$ as a cutoff (Table 2). The clinicopathological factors age, gender, histology were also tested by Cox univariate analysis and had no effect on overall survival. Regarding tumor staging, patients with stage III/IV had a higher risk of death $(\mathrm{HR}=6.21$, $P=0.012)$ as compared to patients with stage I/II.

To obtain a prognostic model independent of staging, a Cox multivariate analysis was performed including the four genes and tumor staging. As a result, three genes, OLR1, CXCL11 and ADAMDEC1, were selected to compose the predictive score, where the risk assigned to each gene in the multivariate analysis was multiplied by the corresponding expression: $z$ score $=(-2.305 \times$ OLR1 expression $)+$ $(-1.936 \times$ CXCL11 expression $)+(-1.768 \times$ ADAMDEC1 expression). The negative value assigned to three genes indicates that higher expression correlates with longer survival. For each patient, a predictive score was calculated and the individual was categorized as high-risk $(-0.27$ to $-2.9)$ or low-risk ( -2.10 to -34.23$)$. New Cox multivariate analysis including only patient staging and the predictive score showed that the high-risk score was an independent prognostic factor (risk of 3.47, $P=0.001$; Table 2).

Although the qRT-PCR validation set of fifty-one samples was not completely independent, including the fifteen samples used in the microarray analyses, the pattern of 
Table 2 Univariate and multivariate analyses of survival (Cox regression)

\begin{tabular}{llll}
\hline Variable & $P$ value & HR & $95 \%$ CI \\
\hline Univariate analysis & & & \\
Age $>66$ years & 0.55 & 1.23 & $0.62-2.41$ \\
Male & 0.75 & 1.11 & $0.56-2.23$ \\
Histologic type diffuse & 0.46 & 0.47 & $0.061-3.52$ \\
Advanced stage (IIIa/IIIb) & $\mathbf{0 . 0 1 2}$ & 6.21 & $1.48-26.0$ \\
CXCL11 negative & $\mathbf{0 . 0 0 9}$ & 2.82 & $1.30-6.10$ \\
OLR1 negative & $\mathbf{0 . 0 0 2}$ & 3.47 & $1.55-7.78$ \\
HLADRB negative & $\mathbf{0 . 0 2 3}$ & 2.31 & $1.12-4.77$ \\
ADAMDEC1 negative & $\mathbf{0 . 0 3 2}$ & 2.13 & $1.07-4.24$ \\
ITGAL negative & 0.051 & 1.99 & $0.98-4.00$ \\
CDC25B negative & 0.068 & 1.89 & $0.90-3.77$ \\
HLADQB negative & 0.18 & 1.59 & $0.80-3.12$ \\
CXCL5 negative & 0.972 & 1.01 & $0.51-1.98$ \\
CADM1 negative & 0.55 & 1.22 & $0.62-2.40$ \\
MUC6 positive & 0.86 & 0.94 & $0.48-1.85$ \\
CLND10 positive & 0.13 & 1.69 & $0.85-3.38$ \\
DAZ positive & 0.14 & 1.69 & $0.85-3.35$ \\
GAST positive & 0.45 & 1.3 & $0.66-2.55$ \\
Multivariate analysis & & & \\
Advanced stage (IIIa/IIIb) & $\mathbf{0 . 0 0 6}$ & 7.63 & $1.79-32.38$ \\
Predictive score of high risk & $\mathbf{0 . 0 0 1}$ & 3.47 & $1.23-5.06$ \\
\hline The & &
\end{tabular}

The statistically significant values are highlighted in bold type

$H R$ hazard ratio, $C I$ confidence interval

expression of OLR1,CXCL11 and ADAMDEC1 among those groups is similar $(P>0.05$, Mann-Whitney test), as shown in supplemental Fig. 4.

Kaplan-Meier analysis showed that patients with a lowrisk predictive score presented with a median survival of 77.0 months, in stark contrast to patients with high-risk score, which showed a 15.8 month median survival $(P=0.003$, Log-rank Test; Fig. $4 c)$.

\section{Dataset searching}

Using the search terms in GEO in September 2013 yielded eight studies that contained "cel." files from either Affymetrix Human Genome U133A or U133 Plus 2.0 Arrays, and contained gene expression profiles from patient tumor samples. Of the eight tumor datasets, only one (GSE29272) contained clinical information of survival for each patient. This series was originally collected to explore the prognostic value of tumor location and contained 134 gastric cancer patients, but survival data were available for 71 gastric noncardia adenocarcinomas (GNCA) and 55 gastric cardia adenocarcinomas (GCA). The normalization by RMA was done using cel. files of 126 tumor samples that were categorized according to patient overall survival, using the cutoff adjusted to 30 months.
The genes related to the biological function "immune response" including CXCL11 and CXCL5, as defined by the FunNet program (GO biological process level 1, supplemental Fig. 5), also showed a strong prognostic value of $p=1.36 \times 10^{-11}$, validating our hypothesis in a larger and independent patient cohort. The gene trio CXCL11 $(P=0.0004)$, ADAMDEC $1 \quad(P=0.0003)$ and OLR 1 $(P=$ not significant $)$ was also partially validated.

As our candidate genes could be expressed by nonimmune cells, an immunohistochemical assay was performed to detect CXCL11 and OLR1 protein expression in tumor samples and non-neoplastic mucosa at the tumor margin in fourteen patients of the identification group.

Non-neoplastic tissue displayed slight reactivity $(1+/$ $4+)$ in fundic glands for CXC11. The lymphocytes and macrophages, usually in small or moderate amounts, were negative or slightly reactive for CXCL11 $(1+/ 4+)$. Other leukocytes were scarce and negative.

Twelve out of 13 samples of gastric adenocarcinoma displayed some reactivity for CXCL11, either at the cytoplasm or at the membrane of epithelial cells. The immunostaining was minimal and focal in nine cases $(1+/ 4+)$ and moderate $(2+/ 4+)$ in three. One case was fully negative. The most relevant finding was the immunoexpression of CXCL11 in intratumoral and peritumoral macrophages in all 12 cases, mostly in moderate amounts $(2+/ 4+$ or $3+/$ $4+)$. In six cases, small amounts of lymphocytes were also found to be reactive $(1+/ 4+)$. Other inflammatory cells and other components of stroma were usually negative. Figure 5 shows the main results for CXCL11.

For OLR1, non-neoplastic stomach at the margins of gastrectomy displayed moderate reactivity $(2+/ 4+$ or $3+/$ $4+)$ in fundic glands. The lymphocytes usually in small or moderate amounts were negative for OLR1. Plasma cells and macrophages were slightly reactive $(1+/ 4+)$. Other leukocytes were scarce and negative. Focal minimal expression was also found at scarce fibroblasts and endothelial cells.

Four out of 12 samples of adenocarcinoma presented some reactivity for OLR1 $(1+/ 4+$ or $2+/ 4+)$. Remarkably, macrophages, plasma cells, fibroblasts and endothelial cells from almost all cases were moderately positive for OLR1 $(2+/ 4+$ or $3+/ 4+)$. Lymphocytes and neutrophils were negative. Figure 6 shows the main results for OLR1 and Supplemental Table 6 shows immunohistochemical assayderived detailed results of CXCL11 and OLR1.

\section{Discussion}

We determined a gene expression profile associated with poor prognosis in a group of patients with gastric adenocarcinoma matched by known prognostic factors. This 

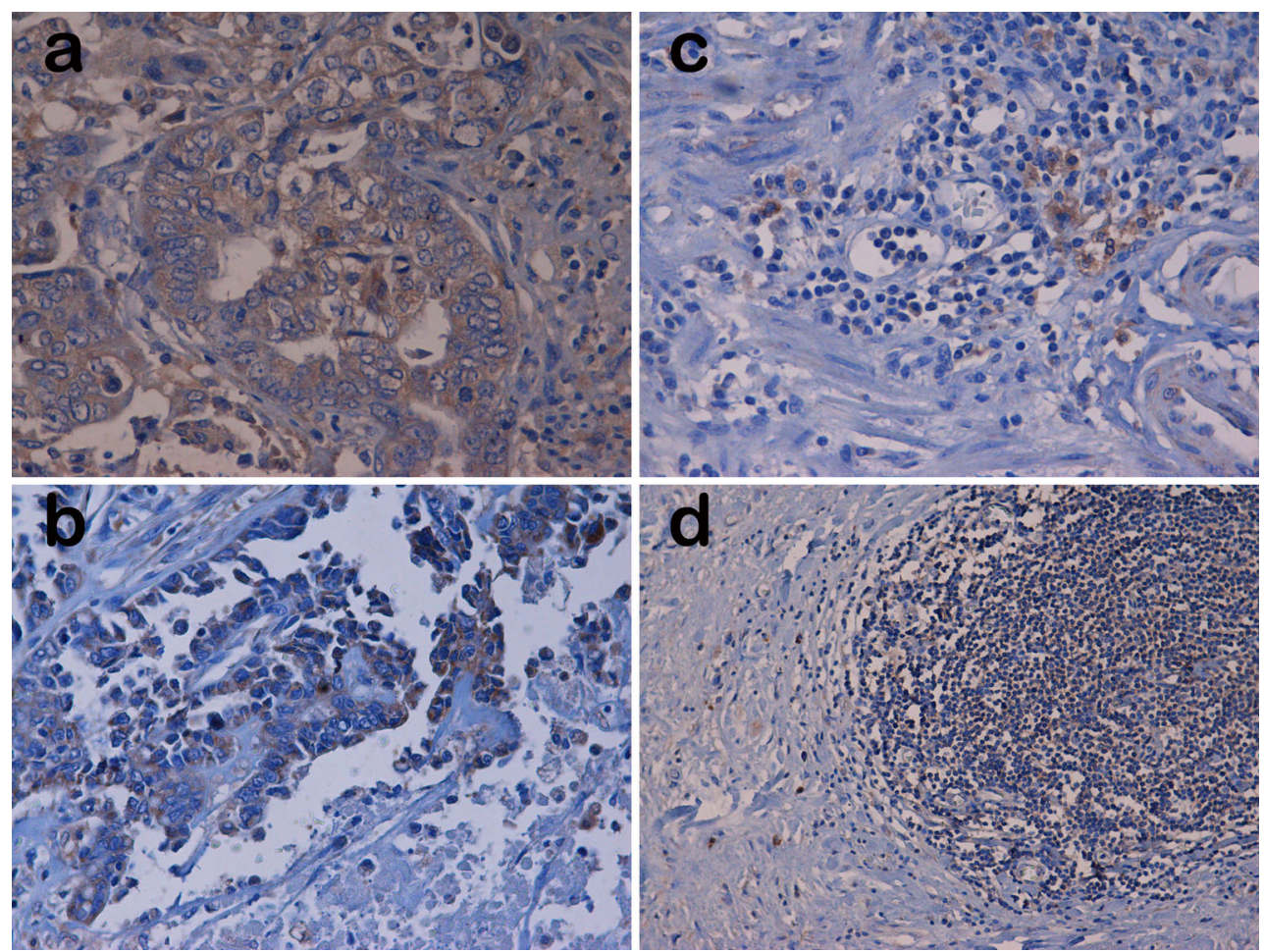

Fig. 5 Immunostaining of $\mathrm{CXC11}$ in gastric adenocarcinoma. a CXCL11 faintly expressed in the cytoplasm of epithelial cells of intestinal-type tubular gastric adenocarcinoma; b Moderate expression in the cytoplasm of epithelial cells of tubule-papillary gastric adenocarcinoma; c Intratumoral inflammatory response: strong

profile is suggestive of immune dampening in the tumor microenvironment. From this profile, a panel of three genes was validated as an independent prognostic factor in a partially separate group of patients. After further validation, this panel may be useful in stratifying patients participating in clinical trials, in order to differentiate risk groups.

In addition, several of these genes are candidates for further functional studies, considering the development of new markers for immunomodulation therapy. This is a highly relevant purpose given that, in general, gastric tumors have a poor prognosis and the only targeted treatment approved for metastatic disease is the antibody trastuzumab, with modest gains in survival when associated with chemotherapy in the management of Her2 overexpressing tumors [13].

Some previously published studies also established gene expression profiles with prognostic value in gastric cancer $[8-10,14,15]$. In a recent study involving 75 patients with adenocarcinoma of the gastroesophageal junction treated by surgery and chemoradiotherapy, a panel of four genes was proposed: deoxycytidine kinase $(D C K), 3$-phosphoadenosine 5-phosphosulfate synthase 2 (PAPSS2), sirtuin 2 (SIRT2), and tripartite motif-containing 44 (TRIM44) [16]. There was no overlap of these genes with those identified macrophage reactivity for CXCL11 $(3+/ 4+)$. Some plasma cells are faintly positive $(1+/ 4+)$, whereas lymphocytes are virtually negative; $\mathbf{d}$ Intratumoral lymphoid aggregate depicting pale reactivity for CXCL11. a-c $\times 400$ and $\mathbf{d} \times 200$

in our study. This discrepancy illustrates the difficulties inherent in this kind of research, which has several sources of heterogeneity: the patients, the tumors and the technical platform used.

We found three lines of evidence favoring immune dampening in the tumor microenvironment of patients with unfavorable prognosis. First, the expression levels of the genes belonging to the functional group of immune/ inflammatory response are markedly reduced as a whole. Second, network analysis suggests an unwired inflammatory response, and third a decreased expression of type 1 helper lymphocyte (Th1) and other immune activating genes was found. Our results are in agreement with other studies. [17-19]. Recently, Jhawer et al. [20] showed that patients with locally advanced gastric tumor, which had perineural invasion and a poor prognosis, also presented a gene expression profile compatible with deregulated immune response. Gastric carcinogenesis is modulated by the immune/inflammatory response triggered by Helicobacter pylori infection [21-23]. The genes that make up our prognostic panel have been previously described as having an important role in tumor biology. ADAMDEC1 (ADAMlike decysin 1) encodes a secreted protein belonging to the disintegrin metalloproteinase family that is expressed in the 

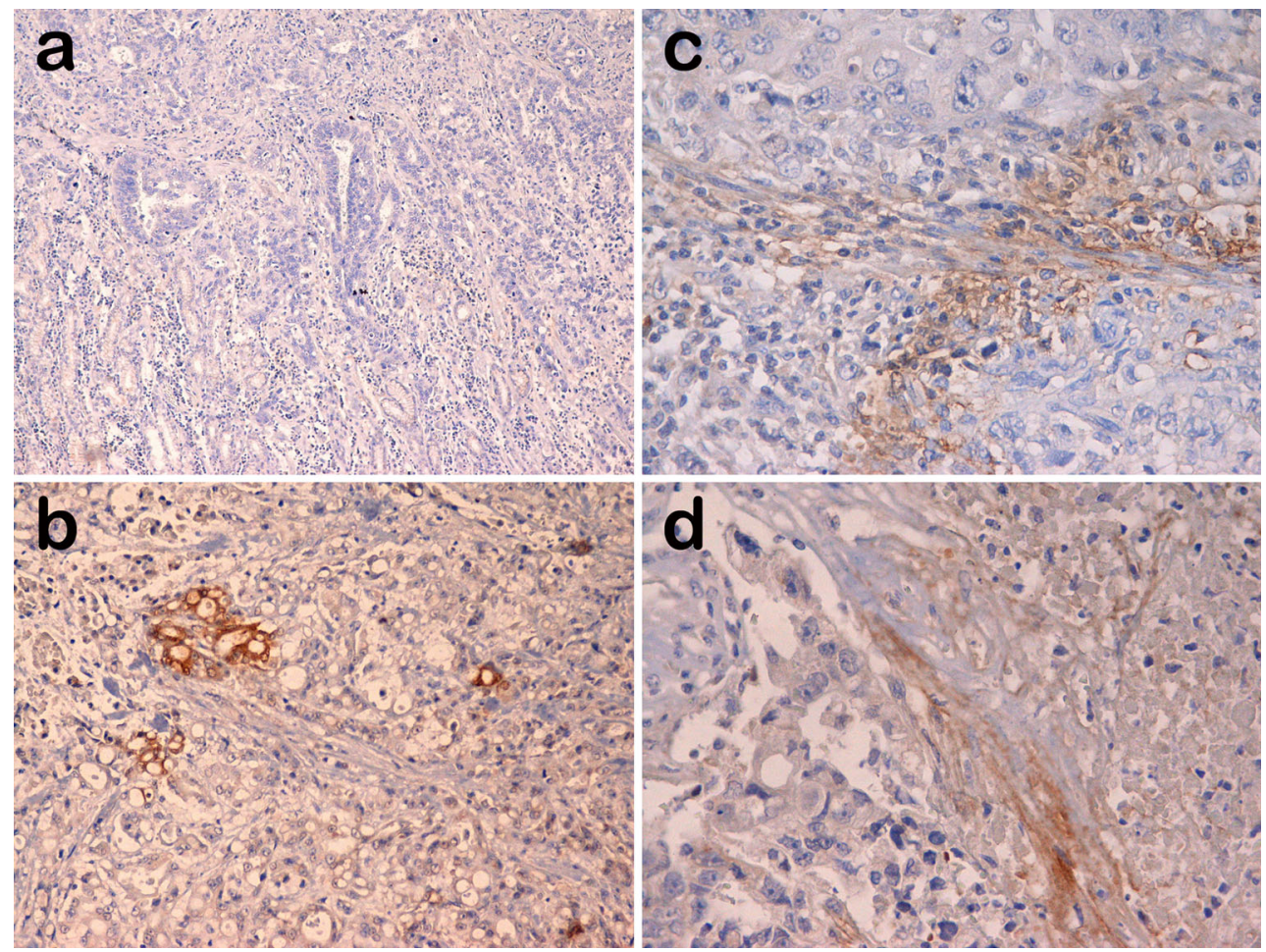

Fig. 6 Immunostaining of OLR1 in gastric adenocarcinoma. a Both tumor and non-neoplastic epithelial cells were negative for OLR1; b Moderate focal positivity for OLR1 in epithelial neoplastic cells; c Positive reaction in intratumoral macrophages, plasma cells and

immune system by macrophages and dendritic cells [24, 25]. Fritsche et al. [26] showed increased expression of $A D A M D E C 1$ during the in vitro differentiation of monocytes into macrophages, and a further increase after classical LPS activation of these macrophages, but no expression in immature DC. In accordance with our data, the expression of $A D A M D E C 1$ at the mRNA and protein level decreased during tumor progression and tumorigenesis of colorectal cancer [27]. Recently, treatment of a human hepatoma cell line (SK-HEP1) with an antimetastatic drug, allyl isothiocyanate (AITC), caused an increase in expression of $A D A M D E C 1$, along with other antimetastatic genes, and inhibited cell migration [28].

CXCL11 [chemokine (CXC motif) ligand 11, I-TAC] encodes a chemokine, which induces a chemotactic response to activated $\mathrm{T}$ lymphocytes, and is the primary ligand for the receptor CXCR-3 that is preferentially expressed on polarized Th1 cells. In renal cell carcinoma, tumor infiltrating lymphocytes have increased expression of $C X C R 3$ and its ligand $C X C L 11$, when compared to peripheral blood $\mathrm{T}$ lymphocytes. In addition, most RCC patients with high expression of CXCL11 showed no recurrence after surgical treatment with curative intent [29], showing a protective effect in these tumors. Furthermore, Quiding-Järbrink et al. [30] showed that gastric macrophages expressed high levels of iNOS and CXCL11, fibroblasts; d Intratumoral fibroblastic reaction displaying moderate reactivity for OLR1, in contrast with epithelial tumor cells which do not express OLR1 in this sample. $\mathbf{a} \times 200$ and $\mathbf{b}-\mathbf{d} \times 400$

indicating a macrophage M1 polarization during $H$ pylori infection.

The OLR1 [Oxidized low-density lipoprotein (lectin-like) receptor 1], or $L O X-1$ gene, encodes a cell membrane receptor responsible for endocytosis and degradation of the oxidized low-density lipoprotein (OxLDL) and was originally described on endothelial cells [31], but is also expressed in smooth muscle cells, dendritic cells and activated macrophages. However, it is not expressed in monocytes and acts as a macrophage scavenger receptor $[32,33]$. This receptor has been associated with various biological functions related to immunity, apoptosis and recruitment of T cells. OLRI is activated in response to oxidized LDL, angiotensin II, TNF- $\alpha$, and other stress stimuli [34], and it is physiologically expressed in cells of innate immunity, such as macrophages, key elements in the polarization and control of immune response. Although a classic Th2 or M2 gene expression panel was not seen, other genes that were associated with unfavorable prognosis in our univariate analysis, such as ITGAL, and MHC class II monomers, have been previously related to T lymphocyte or macrophage polarization. Wang et al. [35] showed that the simultaneously downregulated expression of ITGAL (Integrin, alpha L) and ITGB2 (Integrin, beta 2), and late upregulation of $I L I R 2$ (interleukin 1 receptor, type II) were associated with $\mathrm{T}$ cell activation type II (Th2), a type of response associated with tumor immunosuppression, which is 
Fig. 7 A schematic diagram of gene expression and immune cells that could be associated with poor prognosis of patients with gastric adenocarcinoma (Adapted from Laskin [38]). Genes induced are shown by arrows pointing up and those that were downregulated are indicated by arrows pointing down. Expression data determined by microarray and validated by qPCR assays are indicated by filled arrows and those determined only by microarray assay are indicated by open arrow. Other genes indicated were extracted from literature review. Our data suggest a diminished Th1/M1 polarization and a shift to Th2/ M2 activity, associated with an unfavorable prognosis of gastric cancer

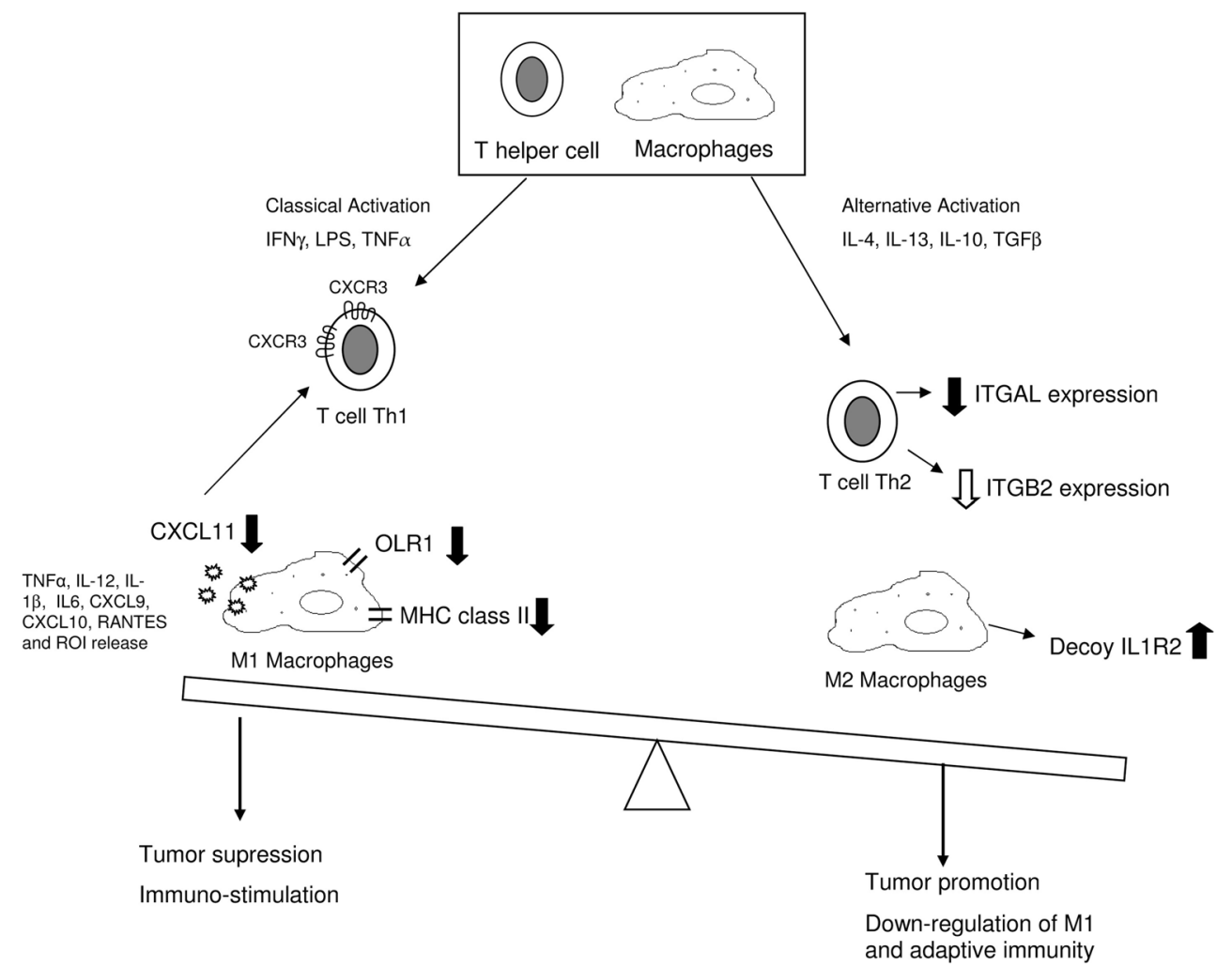

compatible with our results. In gastric carcinoma, the association between MHC class II monomers and $H$. pylori infection has been shown (reviewed by [36]). Moreover, an association between the lack of HLA-DR antigen expression and poor prognosis has also been shown [37].

Our results showed that both CXCL11 and OLR1 proteins were preferentially expressed in the tumor stromal cells, including macrophages, in contrast with the low expression in tumor cells. Taken together, our data indicate that dampened immune/inflammatory response at the tumor microenvironment is associated with poor prognosis. The underlying mechanisms may be related to decreased Th1 activation and possible macrophage alterations (Fig. 7). Those mechanisms merit further studies.

Our study has some strong points. First, all included patients were homogeneously treated in the same institution with strict follow-up. Second, the results are internally consistent: they show that unfavorable prognosis is associated with decreased expression of immune-related genes, to a less coordinated immune response, and to a decreased expression of Th1-related genes, and although our immunohistochemical assay has been performed in a small group of cases, the results were consistent with our hypothesis of CXCL11's and OLR1's association with immune response in the tumor microenvironment. In addition, our hypothesis that immune mechanisms may modulate tumor prognosis is further supported by the in silico validation in a large and totally independent patient cohort. The panel of three genes that were identified as prognostic candidates may be further studied to better understand tumor immune suppression mechanisms, particularly CXCL11 and ADAMDEC1, which were validated in a large and independent cohort.

In conclusion, the trio composed by OLR $1, C X C L 11$ and $A D A M D E C 1$ highlights the attenuation of immune/ inflammatory response in advanced stages of gastric carcinoma. This particular ontology, in general, and especially the trio defined in this study, warrant further investigation as predictive markers in gastric cancer.

Acknowledgments This study was supported by Fundação Faculdade de Medicina and Conselho Nacional de Desenvolvimento Científico e Tecnológico (CNPq no. 577603/2008-6). We thank Mrs. Maria Cristina Piñeiro Grandal for editing the figures, and Mrs Diane D Cohen for additional support.

Conflict of interest The authors declare that they have no conflict of interest.

Open Access This article is distributed under the terms of the Creative Commons Attribution Noncommercial License which permits any noncommercial use, distribution, and reproduction in any medium, provided the original author(s) and the source are credited.

\section{References}

1. Crew KD, Neugut AI. Epidemiology of gastric cancer. World J Gastroenterol. 2006;12:354-62. 
2. Tanigawa N, Amaya H, Matsumura M, Shimomatsuya T. Correlation between expression of vascular endothelial growth factor and tumor vascularity, and patient outcome in human gastric carcinoma. J Clin Oncol. 1997;15:826-32.

3. Chen CN, Hsieh FJ, Cheng YM, Cheng WF, Su YN, Chang KJ, et al. The significance of placenta growth factor in angiogenesis and clinical outcome of human gastric cancer. Cancer Lett. 2004;213:73-82.

4. Mori M, Mimori K, Shiraishi T, Tanaka S, Ueo H, Sugimachi K, Akiyoshi T. p27 expression and gastric carcinoma. Nat Med. 1997;3:593.

5. Akama Y, Yasui W, Yokozaki H, Kuniyasu H, Kitahara K, Ishikawa $\mathrm{T}$, et al. Frequent amplification of the cyclin $\mathrm{E}$ gene in human gastric carcinomas. Jpn J Cancer Res. 1995;86:617-21.

6. Sanz-Ortega J, Steinberg SM, Moro E, Saez M, Lopez JA, Sierra $\mathrm{E}$, et al. Comparative study of tumor angiogenesis and immunohistochemistry for p53, c-ErbB2, c-myc and EGFr as prognostic factors in gastric cancer. Histol Histopathol. 2000; 15:455-62.

7. Zheng L, Wang L, Ajani J, Xie K. Molecular basis of gastric cancer development and progression. Gastric Cancer. 2004;7:61-77.

8. Chen CN, Lin JJ, Chen JJ, Lee PH, Yang CY, Kuo ML, et al. Gene expression profile predicts patient survival of gastric cancer after surgical resection. J Clin Oncol. 2005;23:7286-95. doi:10. 1200/JCO.2004.00.2253.

9. Marchet A, Mocellin S, Belluco C, Ambrosi A, DeMarchi F, Mammano E, et al. Gene expression profile of primary gastric cancer: towards the prediction of lymph node status. Ann Surg Oncol. 2007;14:1058-64.

10. Xu ZY, Chen JS, Shu YQ. Gene expression profile towards the prediction of patient survival of gastric cancer. Biomed Pharmacother. 2010;64:133-9.

11. Vandesompele J, De Preter K, Pattyn F, Poppe B, Van Roy N, De Paepe A, et al. Accurate normalization of real-time quantitative RT-PCR data by geometric averaging of multiple internal control genes. Genome Biol. 2002;3:00341-003411.

12. Pfaffl MW. A new mathematical model for relative quantification in real-time RT-PCR. Nucleic Acids Res. 2001;29:2002-7.

13. Bang YJ, Van Cutsem E, Feyereislova A, Chung HC, Shen L, Sawaki A, et al. Trastuzumab in combination with chemotherapy versus chemotherapy alone for treatment of HER2-positive advanced gastric or gastro-oesophageal junction cancer (ToGA): a phase 3, open-label, randomized controlled trial. Lancet. 2010;376:687-97. doi:10.1016/S0140-6736(10)61121-X.

14. Leung SY, Yuen ST, Chu KM, Mathy JA, Li R, Chan AS, et al. Expression profiling identifies chemokine (C-C motif) ligand 18 as an independent prognostic indicator in gastric cancer. Gastroenterology. 2004;127:457-69.

15. Teramoto K, Tada M, Tamoto E, Abe M, Kawakami A, Komuro $\mathrm{K}$, et al. Prediction of lymphatic invasion/lymph node metastasis, recurrence, and survival in patients with gastric cancer by cDNA array-based expression profiling. J Surg Res. 2005;124:225-36.

16. Peters CJ, Rees JR, Hardwick RH, Hardwick JS, Vowler SL, Ong $\mathrm{CA}$, et al. A 4-gene signature predicts survival of patients with resected adenocarcinoma of the esophagus, junction, and gastric cardia. Gastroenterology. 2010;139:1995-2004.

17. Murata S, Eguchi Y, Terata N, Tani T, Kodama M. Expression of HLA-DR and urokinase-type plasminogen activator in stage IV gastric cancer. Gastric Cancer. 1998;1:71-7.

18. Akaike H, Kono K, Sugai H, Takahashi A, Mimura K, Kawaguchi Y, et al. Expression of high mobility group box chromosomal protein-1 (HMGB-1) in gastric cancer. Anticancer Res. 2007;27:449-57.

19. Wang SK, Zhu HF, He BS, Zhang ZY, Chen ZT, Wang ZZ, et al. $\mathrm{Cag} \mathrm{A}+H$. pylori infection is associated with polarization of $\mathrm{T}$ helper cell immune responses in gastric carcinogenesis. World $\mathrm{J}$ Gastroenterol. 2007;13:2923-31.

20. Jhawer M, Coit D, Brennan M, Qin LX, Gonen M, Klimstra D, et al. Perineural invasion after preoperative chemotherapy predicts poor survival in patients with locally advanced gastric cancer: gene expression analysis with pathologic validation. Am J Clin Oncol. 2009;32:356-62.

21. Nomura A, Stemmermann GN, Chyou PH, Kato I, Perez-Perez GI, Blaser MJ. Helicobacter pylori infection and gastric carcinoma among Japanese Americans in Hawaii. N Engl J Med. 1991;325:1132-6.

22. Parsonnet J, Friedman GD, Vandersteen DP, Chang Y, Vogelman $\mathrm{JH}$, Orentreich $\mathrm{N}$, et al. Helicobacter pylori infection and the risk of gastric carcinoma. N Engl J Med. 1991;325:1127-31.

23. Parkin DM. The global health burden of infection-associated cancers in the year 2002. Int J Cancer. 2006;118:3030-44.

24. Bates EE, Fridman WH, Mueller CG. The ADAMDEC1 (decysin) gene structure: evolution by duplication in a metalloprotease gene cluster on chromosome 8p12. Immunogenetics. 2002;54:96-105. doi:10.1007/s00251-002-0430-3.

25. Shapiro SD. Proteolysis in the lung. Eur Respir J Suppl. 2003;22:30-2.

26. Fritsche J, Muller A, Hausmann M, Rogler G, Andreesen R, Kreutz M. Inverse regulation of the ADAM-family members, decysin and MADDAM/ADAM19 during monocyte differentiation. Immunology. 2003;110:450-7. doi:10.1111/j.1365-2567. 2003.01754.x.

27. Macartney-Coxson DP, Hood KA, Shi HJ, Ward T, Wiles A, O'Connor R, et al. Metastatic susceptibility locus, an 8p hot-spot for tumour progression disrupted in colorectal liver metastases: 13 candidate genes examined at the DNA, mRNA and protein level. BMC Cancer. 2008;8:187-97.

28. Hwang ES, Kim GH. Allyl isothiocyanate influences cell adhesion, migration and metalloproteinase gene expression in SKHep1 cells. Exp Biol Med (Maywood). 2009;234:105-11. doi:10. 3181/0806-RM-190.

29. Kondo T, Nakazawa H, Ito F, Hashimoto Y, Osaka Y, Futatsuyama $\mathrm{K}$, et al. Favorable prognosis of renal cell carcinoma with increased expression of chemokines associated with a Th1type immune response. Cancer Sci. 2006;97:780-6.

30. Quiding-Järbrink M, Raghavan S, Sundquist M. Enhanced M1 macrophage polarization in human Helicobacter pylori-associated atrophic gastritis and in vaccinated mice. PLoS One. 2010;5:15018-27. doi:10.1371/journal.pone.0015018.

31. Sawamura T, Kume N, Aoyama T, Moriwaki H, Hoshikawa H, Aiba Y, et al. An endothelial receptor for oxidized low-density lipoprotein. Nature. 1997;386:73-7.

32. Dunn S, Vohra RS, Murphy JE, Homer-Vanniasinkam S, Walker JH, Ponnambalam S. The lectin-like oxidized low-density-lipoprotein receptor: a pro-inflammatory factor in vascular disease. Biochem J. 2008;409:349-55. doi:10.1042/BJ20071196.

33. Yoshida H, Kondratenko N, Green S, Steinberg D, Quehenberger O. Identification of the lectin-like receptor for oxidized lowdensity lipoprotein in human macrophages and its potential as a scavenger receptor. Biochem J. 1998;334:9-13.

34. Mehta JL, Hu B, Chen J, Li D. Pioglitazone inhibits LOX-1 expression in human coronary artery endothelial cells by reducing intracellular superoxide radical generation. Arterioscler Thromb Vasc Biol. 2003;23:2203-8.

35. Wang M, Windgassen D, Papoutsakis ET. Comparative analysis of transcriptional profiling of CD3+, CD4+ and CD8+ T cells identifies novel immune response players in T-cell activation. BMC Genomics. 2008;9:225-41. doi:10.1186/1471-2164-9-225.

36. McLean MH, El-Omar EM. Genetics of inflammation in the gastrointestinal tract and how it can cause cancer. Recent Results Cancer Res. 2011;185:173-83. 
37. Ma XC, Hattori T, Kushima R, Terata N, Kodama M. Expression of HLA-class II antigen in gastric carcinomas. Its relationship to histopathological grade, lymphocyte infiltration and five-year survival rate. Acta Oncol. 1994;33:187-90.
38. Laskin DL. Macrophages and inflammatory mediators in chemical toxicity: a battle of forces. Chem Res Toxicol. 2009;22:1376-85. 